\title{
Cysteine modification-gated protein dynamics explored using $X-$ ray diffuse scattering
}

\author{
Mark A. Wilson ${ }^{\mathrm{a}}$, Medhanjali DasGupta ${ }^{\mathrm{a}}$, Zhen Su ${ }^{\mathrm{b}}$, Chun Hong Yoon ${ }^{\mathrm{b}}$, Michael \\ E. Wall ${ }^{\mathrm{c}}$, Henry van den Bedem ${ }^{\mathrm{d}}$ \\ a Department of Biochemistry and Redox Biology Center, University of Nebraska, \\ Lincoln, NE 68588; mwilson13@unl.edu \\ binac Coherent Light Source, SLAC National Accelerator Laboratory, Menlo Park, \\ California 94025 \\ ${ }^{\mathrm{C} C o m p u t e r, ~ C o m p u t a t i o n a l, ~ a n d ~ S t a t i s t i c a l ~ S c i e n c e s ~ D i v i s i o n, ~ L o s ~ A l a m o s ~ N a t i o n a l ~}$ \\ Laboratory, Los Alamos, NM 87505 \\ dBioscience Division, SLAC National Accelerator Laboratory, Stanford University, Menlo \\ Park, CA, 94025
}

X-ray diffuse scattering provides information about correlated motions in crystals. Recent advances in detector technology now allow high quality Bragg and diffuse scattering data to be collected simultaneously from protein crystals. These advances have renewed interest in determining if X-ray diffuse scattering can be used to study functionally relevant protein motions. We report preliminary Bragg and X-ray diffuse scattering studies on isocyanide hydratase $(\mathrm{ICH})$, an enzyme involved in the detoxification of isocyanide natural products. Ambient temperature X-ray diffraction shows that $\mathrm{ICH}$ exhibits a correlated displacement of an alpha-helix that occurs as a consequence of radiation-driven oxidation of the active site cysteine residue. X-ray free electron laser (XFEL) serial mix-and-inject crystallography establishes that ICH forms a transient thioimidate intermediate at the active site cysteine residue and that this intermediate triggers similar alpha helical displacement during catalysis. These cysteine modification-gated helical motions provide a way to trigger collective protein motions in a controlled way, making ICH a valuable system for studying diffuse scattering arising from intramolecular correlated motions. Analysis of $\mathrm{ICH}$ X-ray diffuse scattering data suggests that these data may be suitable for more detailed studies of how proteins respond to functionally important perturbations. 